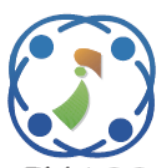

\title{
QALPA- An Efficient QoS Aware Hybrid Localization Model Using Particle Swarm Optimization and Ant Colony Optimization for Cognitive Wireless Sensor Networks
}

\author{
Abhijit Halkai ${ }^{1 *}$ \\ Sujatha Terdal ${ }^{1}$ \\ ${ }^{1}$ Department of Computer Science Engineering, Poojya Dodappa Appa College of Engineering, Kalaburagi, India \\ * Corresponding author's Email: abhijit.halkai@gmail.com
}

\begin{abstract}
A sensor network operates wirelessly and transmits detected information to the base station. The sensor is a small sized device, it is battery-powered with some electrical components, and the protocols should operate efficiently in such least resource availability. Here, we propose a novel improved framework in large scale applications where the huge numbers of sensors are distributed over an area. The designed protocol will address the issues that arise during its communication and give a consistent seamless communication system. The process of reasoning and learning in cognitive sensors guarantees data delivery in the network. Localization in Scarce and dense sensor networks is achieved by efficient cluster head election and route selection which are indeed based on cognition, improved Particle Swarm Optimization, and improved Ant Colony Optimization algorithms. Factors such as mobility, use of sensor buffer, power management, and defects in channels have been identified and solutions are presented in this research to build an accurate path based on the network context. The achieved results in extensive simulation prove that the proposed scheme outperforms ESNA, NETCRP, and GAECH algorithms in terms of Delay, Network lifetime, Energy consumption.
\end{abstract}

Keywords: Sensor, Battery powered, Mobility, Power management, Channel defects, Path selection.

\section{Introduction}

Wireless Communication via sensors has become unavoidable today; it has played an important role in many applications by its sensing and transmitting properties. These sensors need an algorithm with specialized efficient methodologies to achieve efficiency and data delivery to its destination [1]. The sensing and transmitting data wirelessly through connectivity and links in sensors bought the idea of WSNs distributed over an area to monitor the harsh changes in time-critical applications. However, the design constraints such as energy limitation, shortrange communication, less processing power constraints are in need to be incorporated in clustering and routing protocols. Many attempts in literature are made to encounter the limitations but enhancing the quality of service remains challenging. Many attempts towards reducing energy consumption, packet delivery, and packet processing have been proposed [2] which considers many assumptions that may be infeasible in the real world network implementations. Moreover, the adaptability of the sensor at many levels of rigid aspects in the vicinity in terms of dust, vibrations, humidity, temperature, etc. affects the performances[3]; this provides the full or partial changes and failures in the network which forms the major threat in battery depletion, loss of data in WSNs. Sensor protocol needs to be adaptable to the dynamicity in networks such as mobility, communication link failures, channel utilization, retransmissions. Therefore we introduce the energy efficient and delay constraint cognitive aspects of WSN for reliable and successful operations in delay constraint networks. Enhancing the performance of networks remains a challenging process in terms of localization, reliability, network lifetime, etc. There is the prerequisite of proposing a framework in cognitive networks that counts the 
novel cognitive aspects, clustering, and routing in dense and scarce networks for efficient localization. In this paper, we propose the novel hybrid framework enhancing the cognitive nodes for efficient channel utilization, improved PSO when the network is dense and improved ANT when the network is scarce. Efficient data delivery is addressed by efficient channel utilization in cognitive nodes. The two main elements to improvise cognition are; reasoning and learning. The reasoning is utilized to diversify the attributes such as traffic flow, channel availability, energy, power, bandwidth, cluster head election, choosing the path to the destination. reasoning considers short time aspects of the current network. While learning support in obtaining long term goals. The feedback history previously received in messages leads to proactive behaviors in the learning process. The integrated model of cognitive channel utilization and improved PSO for clustering and routing when the network is dense determine the efficient use of resources in large scale applications.

\section{Our contribution to this paper}

We propose a cognitive data delivery approach that addresses the challenges of data delivery in networks comprised of energy-constrained sensors. Here the nodes are built with the cognitive knowledge to share the channels efficiently among the sensors to avoid channel scarcity. Cognitive knowledge provided the awareness to pick the qualified channel for communication from the primary users. Two key elements in cognition are utilized in our approach to implement cognition; reasoning and learning elements. The reasoning is used to differentiate between the attributes of given traffic flow, and choosing the next hop along the data delivery path to the destination. While reasoning realizes short term objectives and makes decisions based on the current network status, learning assists in achieving longterm goals of the network, such as improving its lifetime. The unwanted expenditure of energy in nodes when the density is low is addressed by improved ACO. When the minute mobile nodes concentration in the area of interest is high optimized PSO with cognition is used.

The rest of this paper is discussed as follows. Here, section 2 presents the literature survey of previous works, section 3 presents the proposed design and implementations as a new methodology of this work QALPA, section 4 presents the results and discussion of the proposed QALPA and existing previous work and its relevancies, and the final section 5 concludes about the proposed method and its future needs.

\section{Literature survey}

Wireless sensor networks are the knowledgebased communication networks composed of the numerous sensors that monitor the area and transfers the parametric information anywhere and anytime like vibrations, temperature, humidity, etc. Sensors are severely used in many applications for monitoring, tracking, fire detections, flood detections, etc., and are deployed in very harsh conditions where human reach is impractical. Hence encountering the challenges many research has been carried out in literature encountering issues like coverage, delay constraints, energy conservation, increase in lifetime, connectivity, deployment strategies, etc. [4]. When numerous sensors are deployed far from the end user routing the sensed data towards BS through the shortest path leads to energy conservation. Many routing protocols have been designed and are classified as single-hop and multi-hop protocols [5]. Considering that multi-path does not guarantee the reliability and integrity in the confidential data delivery services, it may lead to packet loss, delay due to congestions, etc. in multi-hop protocols the issue of data redundancy, energy depletion, congestion of numerous packets is addressed and resulted in the formation of clusters among the sensors. The clustering may lead to a decrease in control overhead at sensor levels. LEACH [5] was the first protocol addressed in clustering schemes where clusters are formed in two level phases considering only the energy which may become infeasible in harsh dynamic network conditions. TL-LEACH [6] was proposed, identical to LEACH and considers only the neighboring nodes energy greater than threshold for cluster head selection which forms infeasible for implementation in multi types of sensors. The consideration of parameters distance and residual energy accounting for the role of cluster head GP-LEACH [7]. the non-uniform distribution is addressed in HS-LEACH [8] which again considers only distance and energy. In the HEED [9] protocol, the stochastic selection of clusters is based on node degree and energy which provides better performance than LEACH. With the clustering scheme, unique efficient routing is also very important in the performance of network. As discussed, multi-path routing is efficient with clustering protocols. In review, they reveal the trend alias it as the stochastic protocol with local and random search as the meta-heuristic. The focus of attaining good solutions in the monitoring area is 
carried by local search and explore the possible best solutions [10]. Network Lifetime Enhanced TriLevel Clustering And Routing Protocol(NETCRP) [11]is an application-specific protocol that considers heterogeneity in WSN but fails in considering many aspects in the selection of cluster heads in WSN which results in low efficiency. GA [12] was the first designed algorithm inspired by nature in forming the best solutions by mutation, crossover, and selection. GCA[13] was proposed for clustering which achieves the best solution by considering only the transmission distance and number of cluster nodes. In GAECH [14] the cluster formation is carried by considering only the total energy, standard deviation in a mutation which may imbalance the network, they conclude that GAECH outperforms LEACH and GCA.ACO [15] was proposed where the colony of ants interacts with each other in forming the best optimal solution in search of food. Shortest path to the food was achieved by forming clusters. The arbitrary shaped clusters and problem of outliers from the monitoring area are addressed in ESNA-ACO [16]. PSO [17] is famous among many researchers. The behavior of swarming in particles for optimizing the search solution, speed convergence rate, and easier in selection parameter makes PSO more feasible in wireless sensor networks, the velocity of particles is dependent on position history with cognition. The scheduling in transmission proved in minimal delay, this problem of resource-constrained scheduling is addressed in CPSO. The combination of K-Means and PSO are proposed by [18] on regenerating particles and better explorations. QPSO was proposed for gene expression datasets for clustering and handling an unknown number of clusters [19].Since PSO is very feasible it is extensively used in many applications to enhance network life, energy usage, better coverage, fast convergence, etc. Efficient channel utilization is scarce as the channel available should be used effectively as an elevating demand for WSN. The selection of nodes method for sensing channel in cognitive WSN uses energy efficiency in cooperative sensing. The lifetime maximization is achieved in [20] by using the centralized method for sensing. Fusion Center combines the sensed results from secondary users to optimize sensing result, sensing power by centralized schemes this may be impossible due to communication constraints [21]. To elevate variety of detection in cooperative sensing is achieved by relay selection in CWSN which is a multi-hop sensing scheme which results in more power loss.[22] Proposes the method of sensing by the weight design which uses consensus methods, link failures, practical channel, and channel conditions are utilized in weights.
Load balancing and efficient channel utilization are the main issues to increase the lifetime of networks. Hence form an integrated framework for efficient channel selection and PSO when the densities of the nodes are intense in the specific area and ANT when the network is scarce.

\section{QALPA design and implementation}

\subsection{Network architecture and formation}

The basic structure under context can be summarized as; Sensor Nodes (SNs); which are supposed to be small and have minimal energy with low computational power; hence these nodes can sense data and transmit to the next hop. Cognitive Nodes (CRNs); have more communicating capabilities for efficient channel utilization minimizing the delay. These nodes can be assumed as the main gateway node which forms an intermediate between Base station and sensor nodes. Base station (BS): the main destination where all nodes transmit the data it can control and monitor all the nodes remotely. Considering the universal 802.11 protocol in CRN to provide the Medium Access Control sub layer and a physical layer (PHY) because of its unique feature such as high throughput, low cost, low data rate, less power utilization. Thus, CSMA based 802.11 can be taken into consideration at the MAC layer. The communication channel reliability can be given by

$$
\mathrm{CRN}_{\mathrm{R}}=\left(1-\mathrm{P}_{\mathrm{b}}\right)\left(1-\mathrm{P}_{\mathrm{c} \text {-fail }}\right)\left(1-\mathrm{P}_{\mathrm{p} \text {-dis }}\right)
$$

Where blocking probability because of the buffer full condition is depicted by $\mathrm{P}_{\mathrm{b}}$; Due to channel condition, $\mathrm{P}_{\mathrm{c} \text {-fai }} 1$ presents the channel failure probability. On many attempts of retries, the packets are discarded which is presented as $\mathrm{P}_{\mathrm{p} \text {-di }}$. The sensors $S$ and base station $B_{S}$ in this network are built into a network area. The number of these sensors $S=$ $s_{1} \ldots . s_{n}$ in a network can be increased as much as desired depending on the situation in the area. But the proposed solution addresses the ethics of minimizing the problems and losses caused by the rising number of $S$.

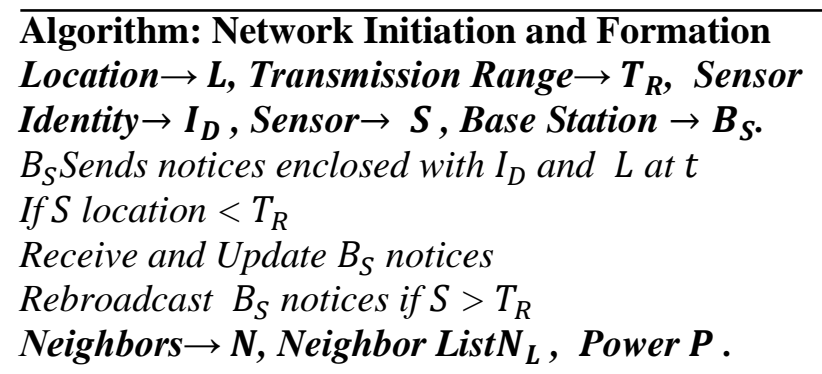


$S$ Sends Hello Message enclosed with

$I_{D}$ and $L$ and $P$ at $t$

If Listening $S$ location $<T_{R}$ $\underline{\text { Update neighbor } N=n_{1} \ldots \ldots n_{n} \text { Each } S \text { prepares } N_{L}}$

Similarly, the $B_{S}$ is located at L. This $B_{S}$ is constantly fencing notices with information about its identity $I_{D}$, and $L$ at regular intervals $t$. Sensors within range of the base station's notification will receive that notification and store the $B_{S}$ identity and $L$. It will then rebroadcast the notification to distant sensors who cannot hear the base station's notification. This way the notification will be sent to all the $S$ in the network. All $S$ are then sent a joining message to the $B_{S}$ along with the sensor $L, I_{D}$ and power $P$. At the same time, each sensor is broadcasting a hello message, this message will be transmitted with the $L$ of the sensor, $P$, and $I_{D}$. This message will go throughout its transmission area $T_{R}$, the transfer area has limitations. With this hello message all the sensors in the network will store information about the neighboring sensor $N=$ $n_{1} \ldots \ldots n_{n}$ in its transmission vicinity and prepare a neighbor list $N_{L}$.

\subsection{Communication model}

During the network lifetime, many aspects may affect the link quality like the location of nodes, antenna shape and orientation, relative distance, radio inference, mobility, and environmental factors. Therefore, the designed protocols should address the changes in link quality and select the efficient path for data delivery. Considering that, changes in the communication channel is varying scale in short range a path loss formula is taken into consideration. The quality aware framework is heavily dependent on the selection of next hops without any delay constraints and minimum energy consumption. The division of bandwidth is done as I sub bands which is converged nearly frequency of $\mathrm{fi}=1,2 \ldots \ldots \mathrm{n}$ and has $\Delta f$ width. The noise p.s.d is accounted as totally flat if the sub band is small enough. If node $\mathrm{j}$ falls in the range of node $i$ with distance $\mathrm{d}_{0}$, the $\mathrm{N}$ hops path endto-end capacity is given by

$$
\mathrm{C}_{\mathrm{EE}}=C_{1}\left(1-\mathrm{CLF}_{\mathrm{AVG}}\right)^{N}
$$

On first hop channel capacity, contribution is given by $C_{1}, \mathrm{~N}$ is the number of hops presented by forwarding schemes, and per hop average capacity loss is given by $\mathrm{CLF}_{\mathrm{AVG}}$.

$$
\operatorname{CLF}_{\mathrm{AVG}}=\operatorname{CLF}\left(\frac{\mathrm{d}_{0}-\mathrm{d}_{\mathrm{AVG}}}{\mathrm{d}_{0}}\right)
$$

$\mathrm{d}_{0}$ Is the distance constant calculated from Euclidean formula, $d_{A V G}$ is given as the average total distance and number of nodes.

\subsection{Energy conservation}

The main aim of designing any of the frameworks in WSN is to conserve available energy in the network. because retaining the energy in networks is impossible in harsh and unreachable environments. The conservation of energy becomes still challenging and never-ending research. $E_{T x}$ is the energy required to transmit packet $\mathrm{P}$ and $E_{R x}$ is the Reception energy need for every packet reception. Hence, energy at the cluster head or Relay node is given by

$$
E_{R N}=L\left(T_{x c} \times E_{T x}+R_{X c} \times E_{R x}+A g_{c} \times E_{A g}\right)
$$

$L$ is the packet Size, $T_{x c}$ is no of packets to be transmitted, $A g_{c}$ is no of packets to be aggregated for forwarding at the relay node, $E_{A g}$ is the energy needed for aggregation.

Cognitive Relay Nodes are assumed to be having higher capabilities when compared to normal Relay nodes. In the process of cognition, the protocol keeps on learning and changing the data path to base station considering energy, reliability, throughput, distance this, increases energy consumption. The energy needed in cognitive Relay Node is given by

$$
\begin{array}{r}
E_{c r n}=L\left(\left(T x_{c} \times E_{T x}+R x_{c} \times E_{R x}+A g_{c} \times\right.\right. \\
\left.\left.E_{A g}\right)\right)+L\left(P_{p} \times\left(E_{C G}-E_{P}\right)\right)(5)
\end{array}
$$

$L$ is the packet size, $T x_{c}, R x_{c}, A g_{c}, P_{p}$ are the number of packets sent, received, aggregated, processed. $E_{R x}, E_{T x}, E_{A g}$ are energy consumed for transmission, reception, and aggregation. The processing overhead during cognition is given by $E_{C G}$ and $E_{P}$

\subsection{Cognitive energy efficient approach (CEEA)}

A novel data delivery approach that is energy aware is proposed here. The gateway node randomly selects sensor $\mathrm{n}$ depending on the performance parameters. $N$ Sensors have many relay nodes in its communication range can be distinguished by spatial Poisson distribution process $X$. Assume the location of the sensor at point a and defined as $L(a, X)$ as the minimum distance from location a to the shortest point of $X$. Such that $L(a, X) \leq r$, only common channels are taken into consideration. Since $X$. is 
spatial poison distribution process $L(a, X) \leq r$, if $R N(d(a, r))>0$, where $d(a, r)$ is radius $r$ centered at $a$. Hence, probability of having only a single relay node in the transmission range of sensor $n$ is presented by;

$$
P(L(a, X) \leq r)=1-\exp \left(A_{d}(d(a, r))\right)
$$

Area of disk $d(a, r)$ is given by $A_{d}$, note that $\exp \left(A_{d}(d(a, r))\right.$ presents no relay node are present in communication range of node n; i.e. the energy and lifetime of neighborhood node $\mathrm{n}$ is lapsed. The cost function for transmission from node $\mathrm{n}$ to $\mathrm{BS}$ is $f\left(n_{j}\right)$ the energy of neighboring node is $e\left(n_{j}\right)$,Euclidean distance to node $\mathrm{n}$ is $d\left(n_{j}\right), i\left(n_{j}\right)$ is initial energy of RN. This approach of cognition has the criteria;1) criteria of evaluation; $f\left(n_{j}\right)=$ $\operatorname{cost}$ (neighbor RN to BS) and $\left.h\left(n_{j}\right)=\min \left(f\left(n_{j}\right)\right), 2\right)$ criteria of selection $e\left(d\left(n_{j}\right)>i\left(d\left(n_{j}\right)\right) \times 50 \%\right)$, $3)$ criteria of termination; no energy is remaining in the range of covered nodes

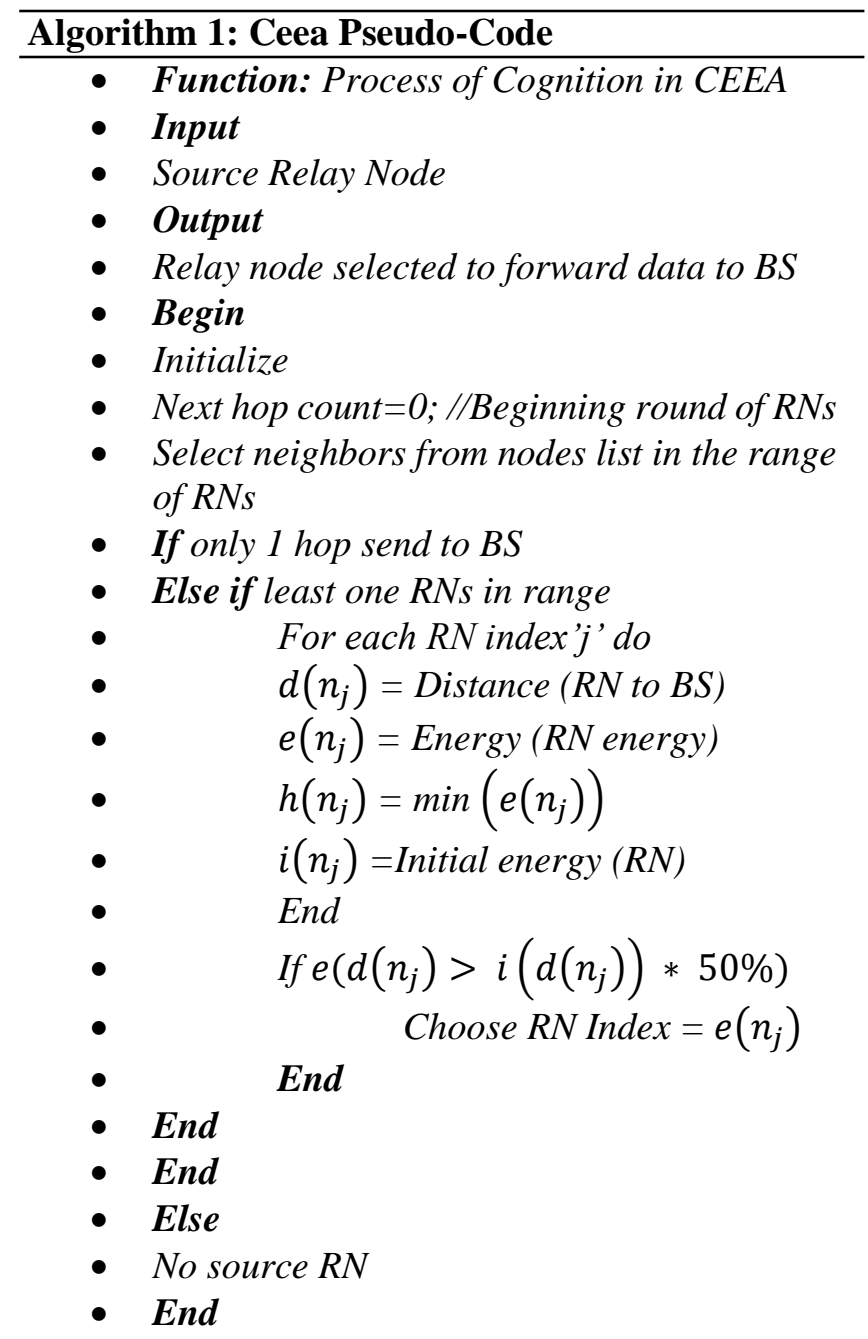

- If RNs Energy <0

- Then terminate from the path

- End

- Update energy distance and coverage

- Criteria of termination

- $P(L(a, X) \leq r)=0$

- End

- Return e $\left(h\left(n_{j}\right)\right)$

Above elements of cognition are utilized in the cognition process in cognitive nodes by reasoning and learning

\section{A. Learning}

To find the appropriate path towards the Base Station, the learning process is used in this approach that satisfies the requirements. A temporary data delivery path is designed through the Cognitive Cluster Node $(\mathrm{CCN})$ based on direction-based heuristics. As the Dynamicity in network topology the aspects of change in location of $\mathrm{CCN}$ each time Cognitive Cluster Node (CSN) has to change the one hop record according to distance, energy aspect and have to choose next hop on every iteration by cognition process. Information of position of CSN and one hop CCN is used as the basic heuristic to form the set of $\mathrm{CCN}$. forwarding the data to the next $\mathrm{CN}$ information is stored for the purpose of next round. Thus, the feedback from previous round and direction-based heuristic helps in cognition process to learn paths of data delivery to sink.

\section{Example 1:}

Assume node $n_{1}$ and $n_{2}$ to transfer data to the sink node $S n_{1}$ and $S n_{1}$, RNs are the intermediate nodes to sink.R5 has the least probability of selection as intermediate as it has the lowest link quality.

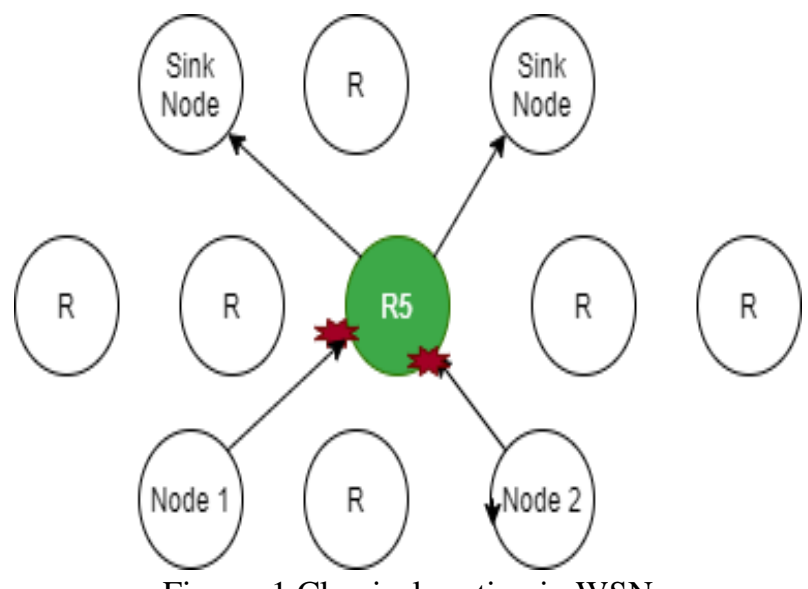

Figure. 1 Classical routing in WSN 


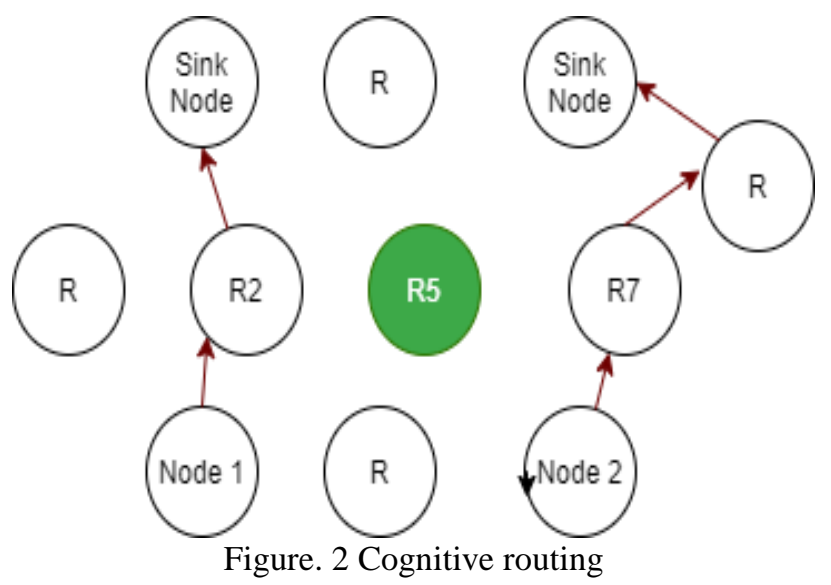

Simultaneous transmissions of data from $n_{1}$ and $n_{2}$ may lead to congestion at R5 due to high traffic as shown in Fig. 1. The approach of learning may detect the congestion on R5 (by the decrease in throughput), proactively pooling this observation with the neighboring nodes, the cognitive network reacts to congestion by providing an alternate path to sink node as shown in Fig. 2.

\section{B. Reasoning}

In this approach, the altered analytic hierarchy process (AHP) is considered for the implementation of reasoning in cognition. Data path is selected on the basis of making multiple criteria decisions. If there exists time sensitive information in path the shortest delay constraint path is chosen avoiding all other aspects provided, it may degrade other metrics such as throughput, lifetime, and energy. If the next two hops provide same delay then other metrics are considered for evaluation and helps in selecting the best node which gives a better performance in long run. Unless there is change in topology the cognition process provides values that are used in the next process hence there is no need to evaluate these values on every transmission rounds. The relative priorities for Relay node selection is given by calculating attributes in Eigen value matrix. The attributes energy, reliability, Throughput, and distance are considered and the next relay is selected with maximum priority root value.

\section{Example 2:}

The main goal is to select the next hop which establishes highest value for that attribute.

Assigning the highest priority to the main attribute energy followed by reliability and throughput, we tabulate relative priorities of these by pair wise comparisons.

1. Form an Eigen matrix of En, Rn, Tn

$$
\mathrm{A}=\left[\begin{array}{ccc}
E n & R n / E n & T n / E n \\
E n / R n & R n / R n & T n / R n \\
E n / T n & R n / T n & T n / T n
\end{array}\right]
$$

2. En is Energy of the node, $\mathrm{Rn}$ is next reliable node, $\mathrm{Tn}$ is throughput calculated as

$$
\text { Tn }=\left(\text { Packet size } \times\left(\text { Ceil }\left(\frac{\text { Distance }}{\text { nodes }}\right)+1\right) \times 8\right)
$$

3. Calculate the Eigen vector of matrix A

4. Distinct roots, Absolute and real values are formed by Eigen vector.

5. Evaluate relative priority values.

\subsection{Cluster selection parameter analysis}

From the above outputs of CEEA algorithm the data critic route is formed through the Relay node. With energy, reliability and throughput, distance it is very essential to select the efficient cluster heads considering the channel utilization technique. Efficient utilization is guaranteed on channel sensing, channel probability, channel decisions, and channel selections based on minimum energy cost, bandwidth and availability ensuring minimum delay in network. Channel Sensing: Each sensor in the list of neighboring calculates the various features given below when trying to select its own $C_{L}$. In addition, the channel $C$ status $S_{C}$ of the network usage will be constantly monitored along with its consistent factors. If $S_{C}$ is good sensor can pick that channel for further computations or else it can skip that channel for that round. We also monitor how much data packets each sensor is able to transmit $P_{S}$ to its next sensor or destination each time it is transmitted, while at the same time how much data packets are lost $P_{L}$ without going to the next node or destination at a time $t$. It was calculated in the manner given below.

$$
\left.S_{C}=\left(X=\sum P_{S}-P_{R}\right), X+\sum P_{L}\right)
$$

If these packet losses are due to an unstable channel and a very large number of data $D$ exchanges arrived $A$ than the channel volume $C_{V}$ that is $A=$ $D$ arrival $>C_{V}$, it may even be due to the lack of space to temporarily store $T_{S}$ the packets in sensor buffer cache $B_{C}$, here $T_{S}=\sum$ D count is $>B_{C} . P_{L}=$ $T_{S}+A+\sum P_{S}$

Channel Probability: Here, channel capacity known as $C_{V}$, it consideration depends on how much data it can send simultaneously, how the sensor and channel handles those packets when more data arrive than their capacity, and what its bandwidth is. 
The congestion between the sensors occurred depends on the parameters given below

- Number of incoming packets

- How to handle incoming packets

- Availability of bandwidth

Highest bandwidth of the channel between $\mathrm{N}$ number of cluster nodes is given by

$$
B=N \times\left(1-\frac{1}{L(\text { Dist-Tdist })+1}\right)
$$

where, $\mathrm{L}$ is the total bytes of packet, $\mathrm{N}$ is no of cluster heads in competition, Dist is intra cluster distance calculated by formula, Tdist is next inter cluster hop distance. Channel congestion $C_{C}$ can occur when too much packets $\sum P_{S}$ are exchanged; it can also lead to packet loss. All of these parameters are monitored in this new protocol as follows.

- The number of times packets are exchanged by a sensor or multiple sensors simultaneously $\sum P_{S}$

- As well as the number of packets lost occurred $\sum P_{L}$ on each sensor connection and the path from start to end

- Between sensors congestion is estimated based on the number of packets that can be stored in the temporary storage area known as cache $B_{C}$ and the number of losses $P_{L}$ that can occur in the channel, here $C_{C}=$ if $\sum\left(T_{S}>B_{C} \& \& P_{L}>C_{V}\right)$.

- These network sensors are movable as we know, in addition, the $D_{\text {ist }}$ between sensors are calculated by Euclidean formula. We saw that it is updated from time to time in the $N_{L}$ of each sensor. This allows us to calculate how many times a sensor is out of touch and reconnected $D_{L}$, this tells the number of times frequent contact deviations occurred due to the movement between two sensors, also this can happen because of the energy loss $P_{C}$ also. $D_{L}=$ $\left(D_{i s t}>T_{R}\right),\left(P_{R}<P\right)$ The number of times contact is missed $D_{L}$. The firmness of the channel $F_{C}$ is calculated by counting the number of times it loses contact $\sum D_{L}$, the number of times it loses data packets because of $\sum C_{C}$, as well as the frequent variations of $S_{C}$, and the sensor buffer overflowing $T_{S}$.

All these activities reduce the number of packets passing through the channel, as well as delaying the flow of packets from a source to destination. This can lead to packet life expiry; $F_{C}$ is therefore considered an important factor in the transmission.

$$
F_{C}=\left(\sum D_{L}, \sum C_{C}, S_{C}, T_{S}\right)>\text { Threshold }
$$

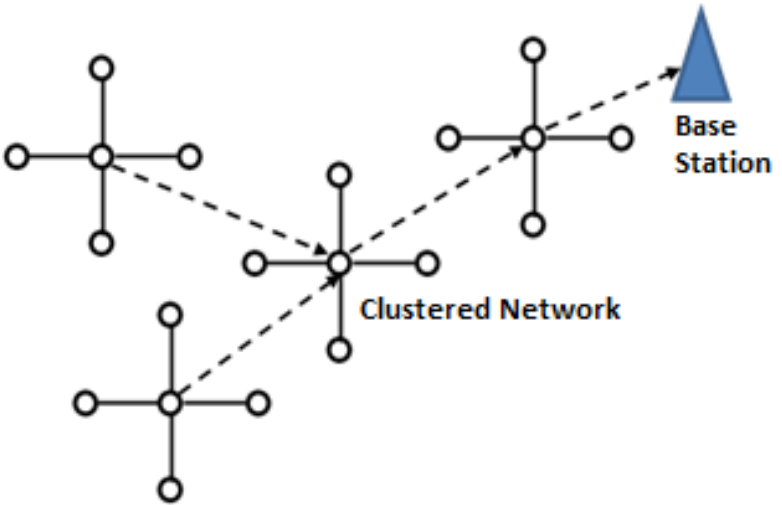

Figure. 3 Cluster formation

We can define if $\sum F_{C}>F_{C}$ unusable channel else it is considered as usable channel for that round. After finalizing all these computations, the path to the $C_{L}$ and from $C_{L}$ to $B_{S}$ is then selected as cluster path from one leader to another from source and total path distance computed as $\sum P D_{i s t}$.

\section{- Channel decision:}

Channel decision is the vital role for selection of efficient delay constraint path towards BS. Proposed algorithm should be capable to choose best available channel to guarantee QOS in WSN. Here, channel decision is based on many parameters in which Energy conservation and delay minimization are the main aspects.

\section{- Channel characterization:}

After the process of channel sensing, determination of the characteristics such as Energy cost, channel probability, energy expenditure, channel interference by delay, congestion etc. are done and taken into consideration for decision process. $E_{C}$ is the energy cost of cognition and is given by

$$
E_{C}=\cos \left(t\left(T_{x c} \times E_{T x}+R_{X c} \times E_{R x}\right)\right)
$$

\section{- Channel selection:}

A best efficient channel is selected on availability based on the above characters. The channel selection criterion is given by:

$$
C_{C D}=E_{c}+\cos (t) \times\left(A g_{c} \times E_{A g}+C_{P} \times E_{E}\right)
$$

$C_{P}$ is channel probability defined above, $E_{E}$ is energy expenditure defined above

\section{- Channel reconfiguration:}

Efficient channel selection algorithm should be able to reconfigure itself with dynamicity of the 
characters. To choose the cluster leader we need to choose the $\sum F_{C}$ sensor along with minimum delay and throughput metrics to act as a $C_{L}$. Using particle swarm technique PSO when the network is overloaded.

\section{Optimized PSO cluster formation}

Particle Swarm Optimization consists of numerous particles $P_{i}, 1<i<\mathrm{N}_{\mathrm{p}}$ travelling with the velocity Each particle has similar dimension $D$ and are assumed to be present at position $X_{i}, 1<\mathrm{d}<\mathrm{D}$. Each particle $P_{i}$ in the network are presented as $P_{i}=$ $X_{1}, X_{2}, X_{3}, X_{4}, \ldots . X_{n}$. The quality of the particle to be selected ad cluster head and next hop node is decided on the fitness function. Until the global best $G_{B e s t}$ is achieved particles follow their own Best $P_{B e s t}$. After every round velocity and $X_{i}$ is with dimension $D$ is updated as

$$
\begin{array}{r}
V_{i}, d(t)=w \times V_{i}, d+\left(g_{1} \times r_{1} \times\left(P_{\text {Best }}-\right.\right. \\
\text { i) } \left.P_{i}\right)+\left(g_{2} \times r_{2} \times\left(G P_{\text {Best }}-\mathrm{i}\right)\right)(14)
\end{array}
$$

$w$ is weight function, $g_{1}$ and $g_{2}$ are positive acceleration factors, $r_{1}$ and $r_{2}$ are random constants.

$$
X_{\text {new }}=X_{\text {old }}+V_{i}, d(t)
$$

\section{Fitness function derivation}

The set of nodes $n_{1}, n_{2}, n_{3}, n_{4}, \ldots . n_{n}$ are assumed to be as the particles. The best efficient node for cluster head selection is dependent of the fitness function. The particle with high fitness is selected as the cluster head. From above CEEA set of parameter schemes are passed on to this Proposed PSO for efficient cluster head selection. Energy cost, channel decision and channel bandwidth are in consideration for fitness function here.

$$
\begin{aligned}
F= & w \times\left(E_{C}+C_{D}+C_{B}\right) \\
& +(1-w)(E+R+T)
\end{aligned}
$$

$\mathrm{W}$ is the weight function constant which is altered for minimizing fitness, where $0<\mathrm{w}<1 . E_{C}$ is energy cost, $C_{D}$ is the channel decided, $C_{B}$ is channel bandwidth, $E$ is the relay energy, $R$ is reliability, $T$ is throughput.

\footnotetext{
PSO for Clustering and Routing

Input: Set of Cluster Nodes $n_{1}, n_{2}, n_{3}, n_{4}, \ldots \ldots n_{n}$, $E_{C}, C_{D}, C_{B}, N e x t \_H o p_{-}, w, g_{1}, g_{2}, r_{1}, r_{2}$

Output: Route R

Step 1: Particles initialization $P_{i}$,

Step 2: from $i-n$ check for covered particles based on distance and range

Step 3: for $1=0$ to $N$
}

$$
\begin{aligned}
& \text { Calculate fitness }\left(P_{i}\right) \\
& P i_{\text {Best }}=P_{i}
\end{aligned}
$$

End for Step 3: $G i_{\text {Best }}\left\{P i_{\text {Best }} \mid\right.$ Fitness $\left(P i_{\text {Best }}\right)=$ min (Fitness $\left.\left(P i_{\text {Best }}\right)\right\}$

Step 4; do

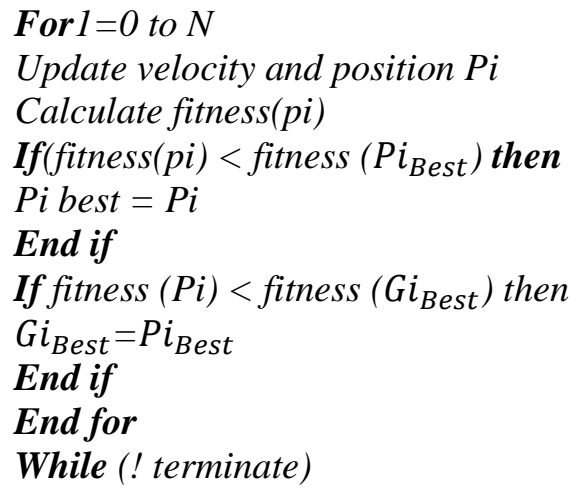

For $1=0$ to $N$

Update velocity and position $\mathrm{Pi}$

Calculate fitness(pi)

If $\left(\right.$ fitness $($ pi $)<$ fitness $\left(P i_{\text {Best }}\right)$ then

Pi best $=P i$

End if

If fitness $(P i)<$ fitness $\left(G i_{\text {Best }}\right)$ then

$G i_{\text {Best }}=P i_{\text {Best }}$

End if

End for

While (! terminate)

End while Calculate the next efficient route repeating all steps

Stop

Select a sensor that effectively handles all of these parameters $E_{C}, C_{D}, C_{B}$, using the particle swarm efficiency method for all parameters that have been monitored and evaluated so far and make it known to area sensors. This PSO method can predict measurements about the $C_{L}$ more accurately than other methods.

\section{Optimized leader and forwarder selection}

Now we first give the input problems to electing the leader as the input of the swarm computation in QALPA protocol as $E_{C}, C_{D}, C_{B}$, which we see as the initial function of this system. Electing a $C_{L}$ for that $T_{R}$ and examining it multiple times as number of iterative process when the network overloaded with sensors. This will renew at each new transmission period starts also observes every action of the object and begins to make a table of its changes. In the sensor that starts sending data packets, $C_{L}$ selects the next $C_{L}$ with the shortest distance $\sum P D_{\text {ist }}$ congestion less channel $C_{L}$ and the least data storage $T_{S}$.

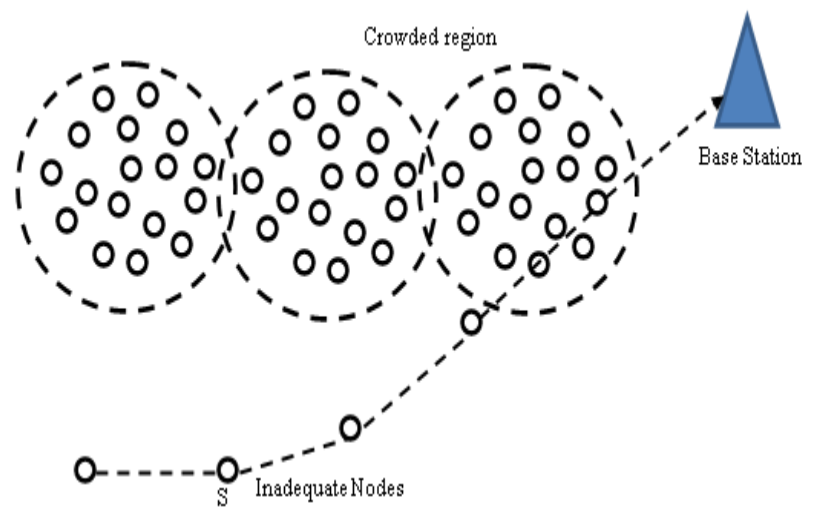

Figure. 4 Localization scheme 


\section{Alternate Path building by ACO}

If the network concentration is scarce all the above schemes are avoided to save unwanted energy consumption. The simple ACO(implemented in ESNA) [16] is considered and the shortest path is formed by high concentration pheromone value of ANTS.

\section{Results and discussions}

The designed protocol is simulated in Network Simulator (NS-2) and output results are compared with our previous work ESNA [16], GAECH [14], and NETCRP [11]. The parameters considered for simulations are given below. The simulation delivers various results which are compared with exiting schemes. Initially 100-200 nodes are considered in 500-500 area with time of simulation as 200s.

Delay in the network may be due to mobility and congestion between channels. In QALPA due to efficient channel, when congestion occurs the transmission channel is changed accordingly for reducing data transmission loss, resulting in minimum congestion. hence, delay of QALPA is less than delay of the other compared protocols. Our proposed Protocol outperforms ESNA by $13 \%$,

Table 1. Simulation parameters

\begin{tabular}{|c|c|}
\hline PARAMETER & \multicolumn{1}{l|}{ VALUE } \\
\hline Targeted Area & $500 \times 500 \mathrm{~m}$ \\
\hline Number Of Nodes & 100 \\
\hline Initial Energy & $100 \mathrm{~J}$ \\
\hline Transmission Energy & $0.02 \mathrm{u} /$ bit \\
\hline Reception Energy & $0.01 \mathrm{u} /$ bit \\
\hline Aggregation Energy & $0.001 \mathrm{uJ} / \mathrm{bit}$ \\
\hline Packet Interval & $0.05 \mathrm{~s}$ \\
\hline $\mathrm{g}_{1}, \mathrm{~g}_{2}, \mathrm{r}_{1}, \mathrm{r}_{2}$ & $0.3,0.2,0.2,0.3$ \\
\hline Packet Size & 512 bytes \\
\hline MAC Protocol & IEEE 802.11 \\
\hline Antenna Model & Omni Antenna \\
\hline
\end{tabular}

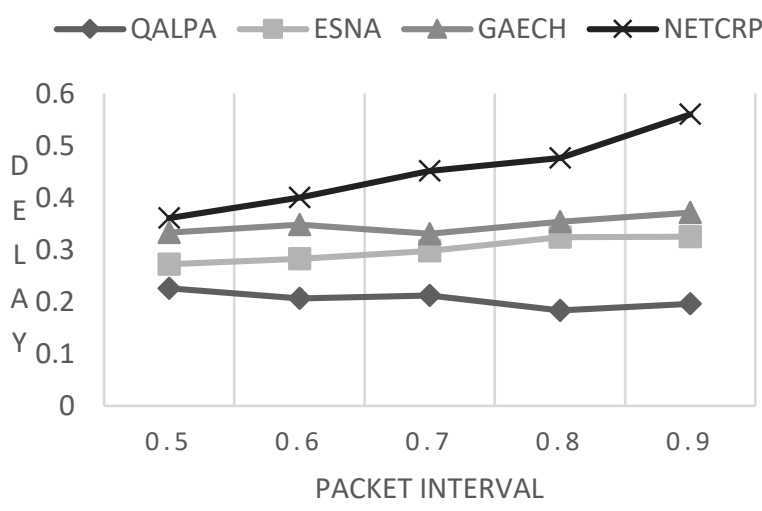

Figure. 5 Packet interval V/S delay

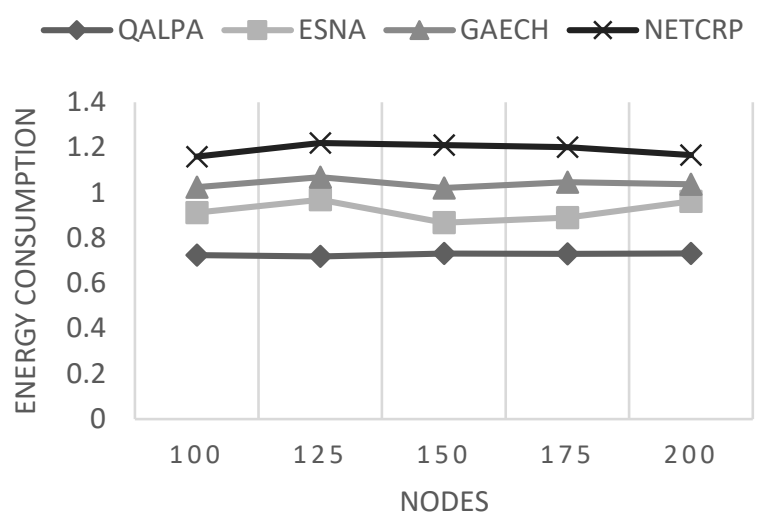

Figure. 6 Node V/S energy consumption

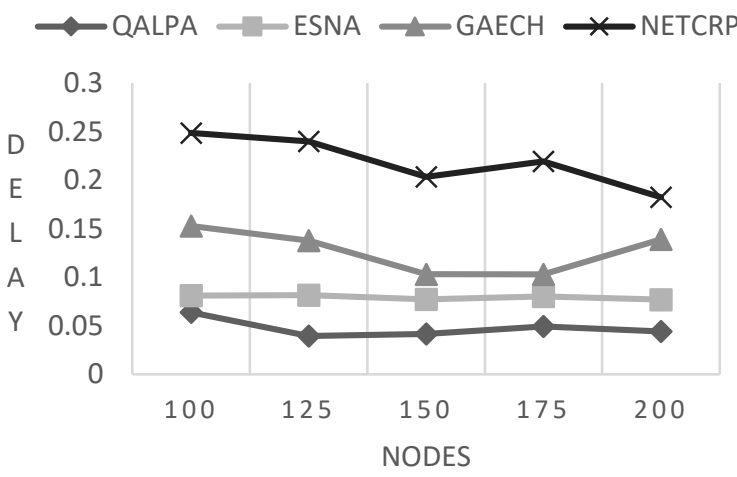

Figure. 7 Node V/S delay

GAECH by $18 \%$ and NETCRP by $36 \%$ at 0.9 as. given in Fig. 5. Efficient cluster head selection is achieved by reasoning and learning in proposed algorithm which lead to decrease in cluster formations and the number of transmissions reduces Due to efficient channel utilization, number of retransmissions due to congestion also reduces resulting in low energy consumption. In the Fig. 6 QALPA has made less power and energy consumption. At the same time other methods have used more power and energy. Our proposed consumes $23.09 \%$ less energy than ESNA, $30.72 \%$ less than GAECH, $43.56 \%$ less than NETCRP. As the number of nodes increases in the network the load and routing path increases.

For successful packet delivery to base station the number of transmission and receptions increases due to network load. The scheme should be reliable enough to handle the network load by increased nodes. Hence, in our proposed scheme the network load is balanced by effective use of PSO and Cognition resulting in outperformance of $16.5 \%$ comparing with ESNA, $47.4 \%$ with GAECH, $69 \%$ with NETCRP.

Network lifetime is total time where in the network is operating and alive. The increase of nodes 


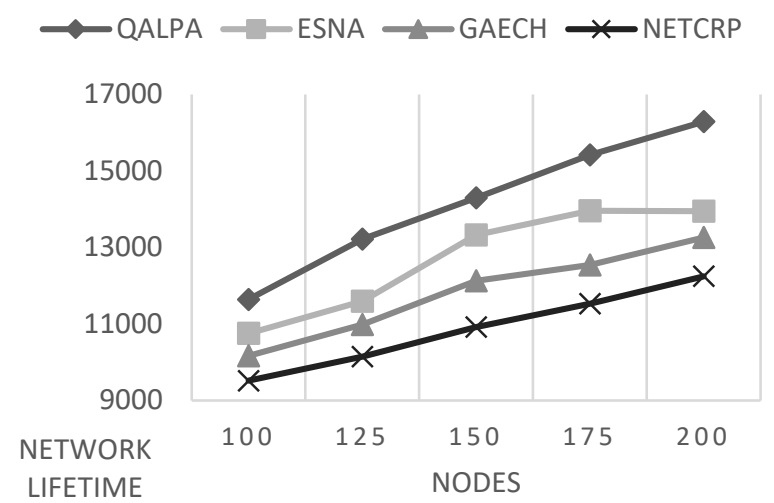

Figure. 8 Nodes V/S Lifetime

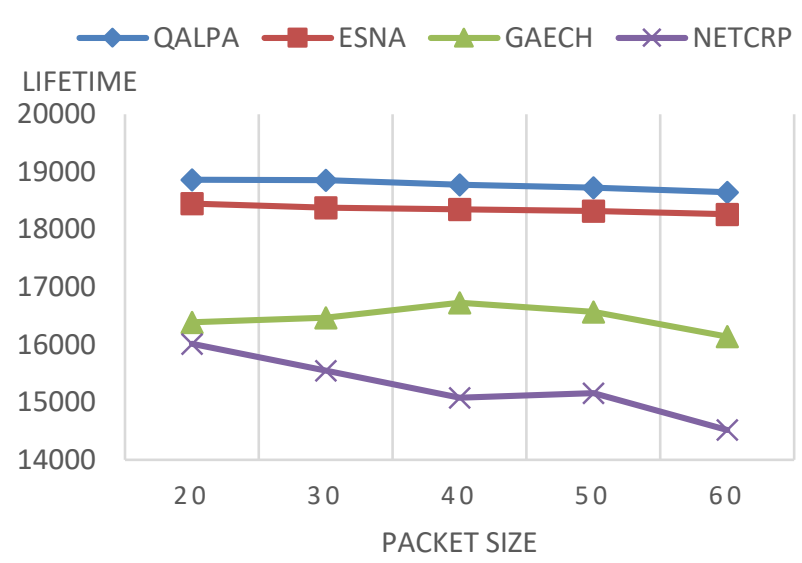

Figure. 9 Packet Size V/S lifetime

in network results in increase of transmissions and receptions in the nodes, resulting in more energy consumption and decrease in total lifetime of network. The efficient usage of channels in our protocol has proved the outperformance compared to existing protocols as given in figure. In Fig. 8 Proposed protocol has $11.3 \%$ more lifetimes compared to ESNA. $14.91 \%$ more than GAECH. $20.22 \%$ more than NETCRP. When the packet size increases the congestion may increase ensuring in increased retransmissions. hence, more retransmissions consume more energy resulting in decrease of lifetime. In our protocol increase in packet size mostly does not affect in lifetime of network. As shown in Fig. 9 the lifetime is $2 \%$ more than ESNA, $13 \%$ more than GAECH, $21 \%$ more than NETCRP.

\section{Conclusion}

In our proposed QALPA, we instigated the clustering and routing protocol for WSN, considering the delay constraints, cognition by learning and reasoning, Efficient channel utilization, dual clustering and routing by improved PSO, with harsh dynamicity of network and energy limitations. First level of clustering is achieved through efficient cognition in WSN by learning and reasoning considering, location changes, minimal distance, energy cost, reliability of covered nodes, throughput. In second level the above all parameters are reinstigated and examined with the efficient channel utilization with, channel sensing, channel probability, channel decision and efficient channel selection by Particle Swarm Optimization. Improved PSO provides the efficient framework for clustering and routing the data towards the Base Station. Our proposed scheme is examined and compared with the previous works ESNA, GAECH, NETCRP. The proposed scheme outperforms ESNA by $13 \%$, GAECH by $25.5 \%$ and NETCRP by $46 \%$ in terms of delay, energy consumption, and network lifetime. In future a new MAC can be designed with new clustering technique

\section{Conflicts of Interest}

With this submission authors confirm no conflict of interest and also confirm that the manuscript has not been published or under consideration else. Thank you for your time and consideration. Any further correspondence can be addressed to abhijit.halkai@gmail.com

\section{Author Contributions}

Abhijit Halkai: Conceptualization, Data curation, Writing- Original draft preparation, Visualization, Investigation Validation, Writing- Reviewing and Editing.

Sujatha Terdal: Supervision, Reviewing.

\section{References}

[1] M. Khalil, A. Khalid, F. U. Khan, and A. Shabbir, "A review of routing protocol selection for wireless sensor networks in smart cities", In: Proc. of 2018 24th Asia-Pacific Conf. Commun. APCC 2018, pp. 610-615, 2019, doi: 10.1109/APCC.2018.8633456.

[2] B. Xue, L. Zhang, W. Zhu, and Y. Yu, "A new sensor selection scheme for Bayesian learning based sparse signal recovery in WSNs", $J$. Franklin Inst., Vol. 355, No. 4, pp. 1798-1818, 2018, doi: 10.1016/j.jfranklin.2017.06.009.

[3] L. Muduli, D. P. Mishra, and P. K. Jana, "Application of wireless sensor network for environmental monitoring in underground coal mines: A systematic review", J. Netw. Comput. Appl., Vol. 106, No. September 2017, pp. 48-67, 2018, doi: 10.1016/j.jnca.2017.12.022.

[4] U. Jain and M. Hussain, "Securing Wireless Sensors in Military Applications through 
Resilient Authentication Mechanism", Procedia Comput. Sci., Vol. 171, No. 2019, pp. 719-728, 2020, doi: 10.1016/j.procs.2020.04.078.

[5] Z. Cui, Y. Cao, X. Cai, J. Cai, and J. Chen, "Optimal LEACH protocol with modified bat algorithm for big data sensing systems in Internet of Things", J. Parallel Distrib. Comput., Vol. 132, pp. 217-229, 2019, doi: 10.1016/j.jpdc.2017.12.014.

[6] S. Arora and S. Singh, "Node Localization in Wireless Sensor Networks Using Butterfly Optimization Algorithm", Arab. J. Sci. Eng., Vol. 42, No. 8, pp. 3325-3335, 2017, doi: 10.1007/s13369-017-2471-9.

[7] H. Peng, Y. Lin, W. Lu, L. Xie, X. Liu, and J. Hua, "Joint resource optimization for DF relaying SWIPT based cognitive sensor networks", Phys. Commun., Vol. 27, pp. 93-98, 2018, doi: 10.1016/j.phycom.2018.02.003.

[8] C. Dong and F. Yu, "A prediction-based asynchronous MAC protocol for heavy traffic load in wireless sensor networks", $A E U$ - Int. J. Electron. Commun., Vol. 82, pp. 241-250, 2017, doi: 10.1016/j.aeue.2017.09.003.

[9] S. K. Susee and B. Chidambararajan, "INFLUENCE OF HIGH-PERFORMANCE ROUTING PROTOCOLS IN WIRELESS SENSOR NETWORKS 652 Figs. 2 and 3 MetaHeuristic Based Approach", No. 3, pp. 651-654, 2019.

[10] D. T. Bui et al., "A novel hybrid approach based on a swarm intelligence optimized extreme learning machine for flash flood susceptibility mapping", Catena, Vol. 179, No. April, pp. 184196, 2019, doi: 10.1016/j.catena.2019.04.009.

[11] D. Agarwal and N. Kishor, "Network lifetime enhanced tri-level clustering and routing protocol for monitoring of offshore wind farms", IET Wirel. Sens. Syst., Vol. 4, No. 2, pp. 69-79, 2014, doi: 10.1049/iet-wss.2013.0050.

[12] S. Prithi and S. Sumathi, "LD2FA-PSO: A novel Learning Dynamic Deterministic Finite Automata with PSO algorithm for secured energy efficient routing in Wireless Sensor Network", Ad Hoc Networks, Vol. 97, p. 102024, 2020, doi: 10.1016/j.adhoc.2019.102024.

[13] S. Bayraklı and S. Z. Erdogan, "Genetic Algorithm Based Energy Efficient Clusters (GABEEC) in Wireless Sensor Networks", Procedia Comput. Sci., Vol. 10, pp. 247-254, 2012, doi: https://doi.org/10.1016/j.procs.2012.06.034.

[14] B. Baranidharan and B. Santhi, "GAECH: Genetic algorithm based energy efficient clustering hierarchy in wireless sensor networks", J. Sensors, Vol. 2015, 2015, doi: $10.1155 / 2015 / 715740$.

[15] L. Panigrahi, "An efficient trust based routing model using ant-colony optimization for the security of WSNs", Int. J. Innov. Technol. Explor. Eng., Vol. 8, No. 8, pp. 1876-1884, 2019.

[16] G. Gajalakshmi and G. Umarani Srikanth, "A survey on the utilization of Ant Colony Optimization (ACO) algorithm in WSN," in 2016 International Conf. on Information Communication and Embedded Systems, ICICES 2016, 2016, doi: 10.1109/ICICES.2016.7518949.

[17] J. R. Parvin and C. Vasanthanayaki, "Particle swarm optimization-based energy efficient target tracking in wireless sensor network", Meas. J. Int. Meas. Confed., Vol. 147, p. 106882, 2019, doi: 10.1016/j.measurement.2019.106882.

[18] A. H. Allam, M. Taha, and H. H. Zayed, "Enhanced Zone-Based Energy Aware Data Collection Protocol for WSNs (E-ZEAL)", $J$. King Saud Univ. - Comput. Inf. Sci., 2019, doi: 10.1016/j.jksuci.2019.10.012.

[19] H. Guo, D. Han, and H. Zhang, "Using PSO to improve ant colony optimization algorithm", IET Conf. Publ., Vol. 2014, No. CP660, pp. 272-276, 2014, doi: 10.1049/cp.2014.1574.

[20] A. H. Gazestani and S. A. Ghorashi, "Distributed Diffusion-Based Spectrum Sensing for Cognitive Radio Sensor Networks Considering Link Failure", IEEE Sens. J., Vol. 18, No. 20, pp. 8617-8625, 2018, doi: 10.1109/JSEN.2018.2866429.

[21] Z. Zhang, X. Wen, H. Xu, and L. Yuan, "Sensing nodes selective fusion scheme of spectrum sensing in spectrum-heterogeneous cognitive wireless sensor networks", IEEE Sens. J., Vol. 18, No. 1, pp. 436-445, 2018, doi: 10.1109/JSEN.2017.2760925.

[22] S. Phoemphon, C. So-In, and D. (Tao) Niyato, "A hybrid model using fuzzy logic and an extreme learning machine with vector particle swarm optimization for wireless sensor network localization", Appl. Soft Comput. J., Vol. 65, pp. 101-120, 2018, doi: 10.1016/j.asoc.2018.01.004. 